\title{
On maximizing clique, clique-Helly and hereditary clique-Helly induced subgraphs
}

\author{
L. Alcón ${ }^{\mathrm{a}, *}$, L. Faria ${ }^{\mathrm{b}}$, C.M.H. de Figueiredo ${ }^{\mathrm{c}}$, M. Gutierrez ${ }^{\mathrm{d}, \mathrm{a}}$ \\ a Departamento de Matemática, Universidad Nacional de La Plata, CC 172, (1900) La Plata, Argentina \\ b Departamento de Matemática, Faculdade de Formaçåo de Professores, Universidade do Estado do Rio de Janeiro, Brazil \\ ' COPPE/Sistemas, Universidade Federal do Rio de Janeiro, Brazil \\ d CONICET, Argentina
}

\section{A R T I C L E I N F O}

\section{Article history:}

Received 3 April 2008

Accepted 6 January 2009

Available online 20 February 2009

\section{Keywords:}

Clique graphs

Clique-Helly graphs

Hereditary clique-Helly graphs

NP-complete

Max SNP-hard

Approximation algorithms

\begin{abstract}
A B S T R A C T
Clique-Helly and hereditary clique-Helly graphs are polynomial-time recognizable. Recently, we presented a proof that the clique graph recognition problem is NP-complete [L. Alcôn, L. Faria, C.M.H. de Figueiredo, M. Gutierrez, Clique graph recognition is NP-complete, in: Proc. WG 2006, in: Lecture Notes in Comput. Sci., vol.4271, Springer, 2006, pp. 269-277]. In this work, we consider the decision problems: given a graph $G=(V, E)$ and an integer $k \geq 0$, we ask whether there exists a subset $V^{\prime} \subseteq V$ with $\left|V^{\prime}\right| \geq k$ such that the induced subgraph $G\left[V^{\prime}\right]$ of $G$ is, variously, a clique, clique-Helly or hereditary cliqueHelly graph. The first problem is clearly NP-complete, from the above reference; we prove that the other two decision problems mentioned are NP-complete, even for maximum degree 6 planar graphs. We consider the corresponding maximization problems of finding a maximum induced subgraph that is, respectively, clique, clique-Helly or hereditary cliqueHelly. We show that these problems are Max SNP-hard, even for maximum degree 6 graphs. We show a general polynomial-time $\frac{1}{\Delta+1}$-approximation algorithm for these problems when restricted to graphs with fixed maximum degree $\Delta$. We generalize these results to other graph classes. We exhibit a polynomial 6-approximation algorithm to minimize the number of vertices to be removed in order to obtain a hereditary clique-Helly subgraph.
\end{abstract}

(c) 2009 Elsevier B.V. All rights reserved.

\section{Introduction}

A complete set of a graph $G=(V . E)$ is a subset of $V$ inducing a complete subgraph. A clique is a maximal complete set. Denote by $C(G)$ the clique family of $G$. The clique graph $K(G)$ of $G$ is the intersection graph of $C(G)$. Say that $G$ is a clique graph if there exists a graph $H$ such that $G=K(H)$. A clique-Helly graph is a graph where $C(G)$ satisfies the Helly property: any pairwise intersecting subfamily of $C(G)$ has non-empty total intersection [1]. A hereditary clique-Helly graph is a graph where every induced subgraph is clique-Helly. The class of hereditary clique-Helly graphs $(\mathrm{H} \mathcal{K} H$ ) is contained in the class of clique-Helly graphs $(\mathcal{K H})$, which in turn is contained in the class of clique graphs $(\mathcal{K})$.

Clique graphs and subclasses have been much studied as intersection graphs, in the context of graph operators, and are included in several books [2-5].

\footnotetext{
5t An extended abstract of this paper was presented at LAGOS 2007, the IV Latin-American algorithms, graphs and optimization symposium, and appeared in Electronic Notes in Discrete Mathematics 30 (2008) 147-152. This research was partially supported by CNPq, Prosul-CNPq, CAPES (Brazil)/COFECUB (France), Prociência Project - FAPERJ.

* Corresponding author. Tel.: +54 2214245875 ; fax: +542214229850.

E-mail addresses: liliana@mate.unlp.edu.ar (L. Alcỏn), luerbio@cos.ufrj.br (L. Faria), celina@cos.ufrj.br (C.M.H. de Figueiredo), marisa@mate.unlp.edu.ar (M. Gutierrez).
} 
Let $A$ represent a class of graphs; consider the following problems:

A-RECognition (A-REC)

Instance: Graph $G=(V, E)$.

Question: Does $G$ belong to $A$ ?

A-SUbGRAPH (A-SUB)

Instance: Graph $G=(V, E)$ and a positive integer $k$.

Question: Is there a subset $V^{\prime} \subseteq V$ with $\left|V^{\prime}\right| \geq k$ such that the subgraph $G\left[V^{\prime}\right]$ induced by the set $V^{\prime}$ belongs to $A$ ?

MAXIMUM A-SUBGRAPH $($ MAX-A)

Instance: Graph $G=(V, E)$.

Goal: Find a maximum subset $V^{\prime} \subseteq V$ such that the subgraph $G\left[V^{\prime}\right]$ induced by the set $V^{\prime}$ belongs to $A$.

Clearly, if A-REC is an NP-complete problem, then A-SUB is also an NP-complete problem. Moreover, $A$-SUB is NP-complete for any polynomial-time recognizable class of graphs A which is hereditary, contains arbitrarily large graphs and is not the class of all graphs [6]. Examples of such classes are hereditary clique-Helly, comparability, permutation, perfect, circular-arc, circle, line, planar, bipartite, chordal and interval graphs. However, there are some important - of course non-hereditary - classes of graph for which both A-REC and A-SUB are polynomial. Instances are the class of connected graphs and the class of graphs with a perfect matching. Consequently, studying the complexity of A-SuB is not trivial. In addition, when A-SUB is NP-complete, there is no polynomial-time algorithm for resolving MAX-A (providing that $\mathrm{P} \neq \mathrm{NP}$ ); so it is natural to ask for approximation algorithms for MAX-A.

In this paper we study those problems for the classes $\mathrm{H} \mathcal{K} H, \mathcal{K} \mathrm{H}$ and $\mathcal{K}$.

In [7,8], it was shown that $\mathrm{H} \mathcal{K} H$-REC and $\mathcal{K} H$-REC are polynomial-time problems. In [9], we presented a proof that $\mathcal{K}$-REC is NP-complete, and thus $\mathcal{K}$-suB is NP-complete. In Section 3, we prove that both $\mathrm{H} \mathcal{K} \mathrm{H}$-suB and $\mathcal{K} \mathrm{H}$-suB are NP-complete for maximum degree 6 planar graphs. We also prove that MAX-H $\mathcal{K} \mathrm{H}, \mathrm{MAX}-\mathcal{K} \mathrm{H}$ and MAX- $\mathcal{K}$ are Max SNP-hard for maximum degree 6 graphs, meaning that [10] they are approximable with a fixed ratio in polynomial time but [11] there is a constant $\varepsilon>0$ such that the existence of a polynomial-time approximation algorithm for MAX-H $\mathcal{K} H$, for MAX- $\mathcal{K} H$, or MAX- $\mathcal{K}$, restricted to maximum degree 6 graphs, with performance ratio at most $1+\varepsilon$, implies that $\mathrm{P}=\mathrm{NP}$.

In Section 4 , we present a general result giving necessary conditions for a class of graphs $\mathcal{A}$, characterized by a collection of forbidden induced subgraphs, to satisfy the property that MAX-A is Max SNP-hard.

Besides the negative result of Section 3, we show in Section 5 two simple polynomial-time approximations: a $\frac{1}{A+1}-$ approximation algorithm for the problems MAX-H $\mathcal{K} \mathrm{H}, \mathrm{MAX}-\mathcal{K} \mathrm{H}$ and MAX- $\mathcal{K}$ restricted to graphs with bounded degree $\Delta$; and a 6 -approximation algorithm to minimize the number of vertices to be removed in order to obtain a hereditary cliqueHelly subgraph.

\section{Preliminaries}

Our complexity results are supported by reductions from the NP-complete [12] problem VERTEx covER (VC) for planar cubic graphs, and the Max SNP-hard $[13,11,10]$ problem MiNIMUM VERTEX Cover (MIN-VC) for planar cubic graphs, defined next.

VERTEX COVER (VC)

Instance: Planar cubic graph $G=(V, E)$ and a positive integer $k$.

Question: Is there a subset $V^{\prime} \subseteq V$ such that $\left|V^{\prime}\right| \leq k$ and, for every edge $u v \in E$, $u$ or $v$ belongs to $V^{\prime}$ ?

MINIMUM VERTEX COVER (MIN-VC)

Instance: Planar cubic graph $G=(V, E)$.

Goal: Find a minimum $V^{\prime} \subseteq V$ such that for every edge $u v \in E, u$ or $v$ belongs to $V^{\prime}$.

For establishing that a problem is Max SNP-hard, we use the approximation preserving reduction, called $L$-reduction, of Papadimitriou and Yannakakis [10].

Let $\mathbf{A}$ and $\mathbf{B}$ be two optimization problems. We say that $\mathbf{A}$ L-reduces to $\mathbf{B}$ if there are two polynomial-time algorithms $f$ and $g$ and positive constants $\alpha$ and $\beta$ such that for each instance $I$ of $\mathbf{A}$ :

(1) Algorithm $f$ produces an instance $I^{\prime}=f(I)$ of $\mathbf{B}$ such that the optima of $I$ and $I^{\prime}$ satisfy $O p t_{\mathbf{B}}\left(I^{\prime}\right) \leq \alpha O p t_{\mathbf{A}}(I)$.

(2) Given any feasible solution of $I^{\prime}$ with cost $c^{\prime}$, algorithm $g$ produces a feasible solution of $I$ with cost $c$ such that $\left|c-O p t_{\mathbf{A}}(I)\right| \leq \beta\left|c^{\prime}-O p t_{\mathbf{B}}\left(I^{\prime}\right)\right|$.

The main point about L-reductions is that if problem $\mathbf{A}$ L-reduces to problem $\mathbf{B}$ and problem $\mathbf{B}$ allows an $\varepsilon$-approximation algorithm, then problem $\mathbf{A}$ allows an $(\alpha \beta \varepsilon)$-approximation algorithm.

\section{Clique, clique-Helly and hereditary clique-Helly classes}

A polynomial-time recognition algorithm for the class of hereditary clique-Helly graphs was presented in [7]. This algorithm uses a finite family of forbidden induced subgraphs, the so-called ocular graphs depicted in Fig. 1. In [7], ocular 


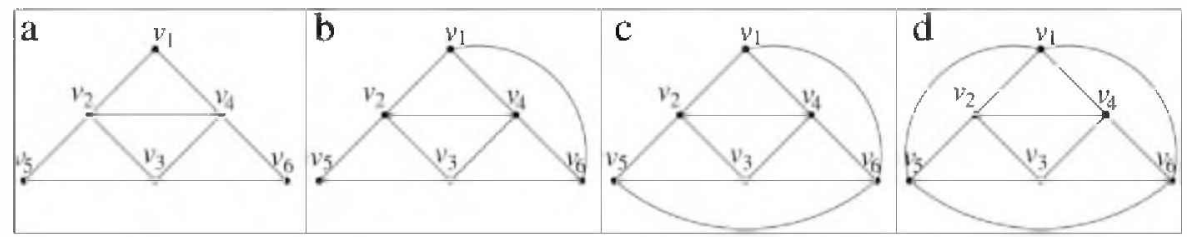

Fig. 1. Ocular graphs: minimal forbidden configurations for hereditary clique-Helly graphs.
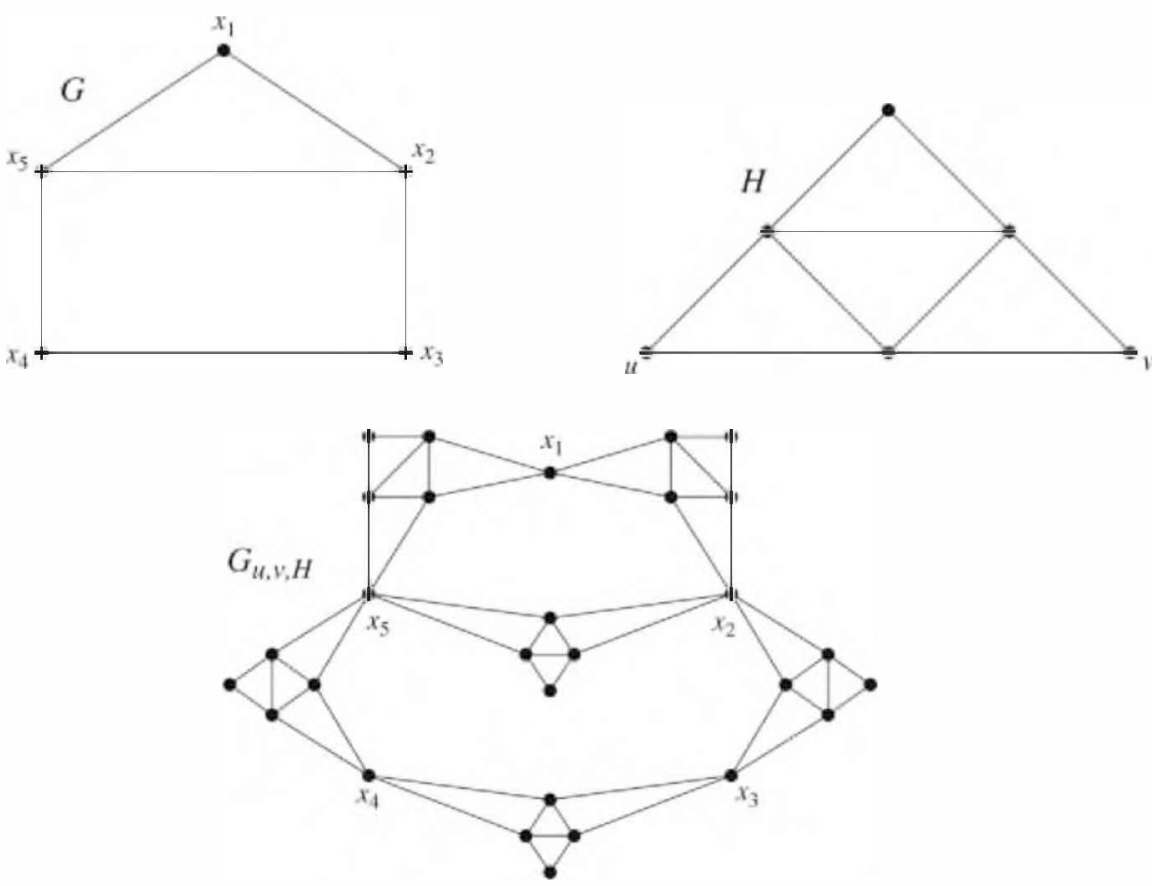

Fig. 2. Graph $G_{y, H}$ obtained by replacing each edge of $G$ by a Hajos graph.

graphs were shown to be the minimal forbidden configurations for hereditary clique-Helly graphs. The graph $H$ in Fig. 2 , called the Hajos graph, is one of the ocular graphs.

For $K_{4}$-free graphs, the classes of clique graphs, clique-Helly graphs and hereditary clique-Helly graphs are the same [5].

Let $G=(V, E)$ be a graph. Let $H$ be the Hajós graph and $u, v$ be the two non-adjacent vertices of $H$ depicted in Fig. 2 . We obtain a new graph $G_{u, v, H}$ by replacing each edge of $G$ by a Hajos graph. This means that $G_{u, v, H}$ is the graph where $V(G) \subseteq V\left(G_{u, v, H}\right)$ and, for each edge $x y$ of $G$, there is a copy $S_{x y}$ of $H-\{u, v\}$ in $G_{u, v, H}$, and the additional set of edges $E_{x y}=\left\{x s: s \in V\left(S_{x y}\right)\right.$ and $\left.u s \in E(H)\right\} \cup\left\{y t: t \in V\left(S_{x y}\right)\right.$ and $\left.v t \in E(H)\right\}$.

We give an example for this operation in Fig. 2.

Lemma 1. Let $G=(V . E)$ be any graph and $G_{u, v, H}$ be the graph obtained from $G$ by the process described above. Then:

1. $G_{u, v, H}$ is $K_{4}$-free.

2. If $G$ is cubic and planar, then $G_{u, v, H}$ is a maximum degree 6 planar graph.

3. For any edge xy of $G$, the subgraphs of $G_{u, v, H}$ induced by $V\left(S_{x y}\right) \cup\{x, y\}$ are isomorphic to the Hajos graph $H$. These are the only induced subgraphs of $G_{u, v_{H}}$ that are isomorphic to an ocular graph.

4. $\left|V\left(G_{u, v, H}\right)\right|=|V|+4|E|$.

5. If $V^{\prime} \subseteq V$, then $V$ is a vertex cover of $G$ if and only if $G_{u, v, H}-V$ is an ocular-free induced subgraph of $G_{u, v, H} . M o r e o v e r V$ is minimum if and only if $G_{u, v, H}-V$ has the largest number of vertices.

Proof. The four first items are easily verifiable.

Let $V \subseteq V$. By item $3, V$ is a vertex cover of $G$ if and only if $G_{u, v, H}-V$ is an ocular-free induced subgraph of $G_{u, v, H}$. It follows that if $G_{u, v, H}-V^{\prime}$ is maximum then $V^{\prime}$ is minimum.

Let us prove the reciprocal implication. Assume $V^{\prime}$ is a minimum vertex cover of $G$. We want to show that the ocularfree subgraph $G_{u, v, H}-V^{\prime}$ of $G_{u, v, H}$ is a maximum ocular-free induced subgraph, i.e. has the largest number of vertices. Let $S=G_{u, v, H}-T, T \subseteq V\left(G_{u, v, H}\right)$, be any maximum ocular-free induced subgraph of $G_{u, v, H}$. Suppose there exists an edge $x y$ of $G$ such that both end vertices, $x$ and $y$, belong to $V(S)=V\left(G_{u, v, H}\right)-T$. Since $S$ is ocular-free, by 3 , at least one vertex $h$ of $S_{x y}$ 
is not in $V(S)$, i.e. $h \in T$. Then by removing $h$ from $T$ and by adding $x$ to $T$, a new ocular-free subgraph of $G_{u, v, H}$ with $|V(S)|$ vertices is obtained.

It follows that there exists $S^{\prime}=G_{u, v, H}-T^{\prime}$, a maximum ocular-free induced subgraph of $G_{u, v, H}$ such that at least one end vertex of each edge of $G$ belongs to $T^{\prime}$. Thus $T^{\prime}$ contains a vertex cover of $G$ and so $\left|T^{\prime}\right| \geq\left|V^{\prime}\right|$, and so $\left|V\left(G_{u, v, H}-V^{\prime}\right)\right| \geq$ $\left|V\left(G_{u, v, H}-T^{\prime}\right)\right|$. Then $G_{u, v, H}-V^{\prime}$ is maximum.

Theorem 2. $\mathcal{K} H$-suB and $\mathrm{H} \mathcal{K} H$-suB are NP-complete problems for maximum degree 6 planar graphs.

Proof. Since $\mathcal{K} \mathrm{H}(\mathrm{H} \mathcal{K} H)$ is in $\mathrm{P}$, then $\mathcal{K}$ H-sub ( $\mathcal{K} \mathcal{K} H$-suB) is in NP.

We are going to reduce vc restricted to planar cubic graphs to $\mathcal{K}$ H-SUB ( $\mathrm{H} \mathcal{K} H$-SUB). Let $(G, k)$ be an instance of vc where $G=(V, E)$ is a planar cubic graph and $k$ is a positive integer. We define in polynomial time, on the size of $G$, using the described operation, the graph $G_{u, v, H}$ and the integer $k^{\prime}=|V|+4|E|-k$ as an instance of $\mathcal{K}$-sUB (H $\mathcal{H} H$-sUB), restricted to maximum degree 6 planar graphs, by Lemma 1 item 2 .

We claim that there exists a vertex cover of $G$ of size at most $k$ if and only if there exists an ocular-free induced subgraph of $G_{u, v, H}$ of size at least $k^{\prime}$; since $G_{u, v, H}$ is $K_{4}$-free (Lemma 1 item 1 ), it happens if and only if there exists a $\mathcal{K H}(\mathrm{H} \mathcal{K} H)$ induced subgraph of $G_{u, v, H}$ of size at least $\boldsymbol{k}^{\prime}$. Thus the proof follows.

Indeed, there exists a vertex cover of $G$ of size at most $k$ if and only if there is $V^{\prime}$ minimum vertex cover of $G$ with $\left|V^{\prime}\right| \leq k$. From Lemma 1 item $5, V^{\prime}$ is a minimum vertex cover of $G$ if and only if $G_{u, v, H}-V^{\prime}$ is a maximum ocular-free induced subgraph. The size of $G_{u, v, H}-V^{\prime}$ is $\left|V\left(G_{u, v, H}-V^{\prime}\right)\right|=\left|V\left(G_{u, v, H}\right)\right|-\left|V^{\prime}\right|$. By Lemma 1 item 4, this is equal to $|V|+4|E|-\left|V^{\prime}\right| \geq|V|+4|E|-k=k^{\prime}$.

\section{Theorem 3. MAX- $\mathcal{K}$, MAX- $\mathcal{K} H$ and MAX-H $\mathcal{K} H$ are Max SNP-hard for maximum degree 6 graphs.}

Proof. We L-reduce the Max SNP-hard problem MIN-vc restricted to planar cubic graphs $[13,11,10]$ to MAX- $\mathcal{K}$, MAX- $\mathcal{K H}$ and MAX-H $\mathcal{K H}$. The algorithm $f$ of the L-reduction is the one which obtains the graph $G_{u, v, H}$ from a graph $G=(V, E)$. As in the proof of the previous theorem, since $G_{u, v, H}$ is $K_{4}$-free, the three problems, MAX- $\mathcal{K}$, MAX- $\mathcal{K} \mathrm{H}$ and MAX-H $\mathcal{K} \mathrm{H}$, are equivalent to maximum ocular-free induced subgraph.

We observe that as $G$ is a cubic graph, then $|E|=\frac{3}{2}|V|$. Consider $V^{\prime}$ a minimum vertex cover of $G$, and let $E^{\prime}$ be the set of edges of $G$ with exactly one endpoint in $V^{\prime}$. Remark that $V \backslash V$ is an independent set and as $G$ is cubic we have that $3\left|V \backslash V^{\prime}\right|=\left|E^{\prime}\right| \leq 3\left|V^{\prime}\right|$. Hence, $|V|=\left|V \backslash V^{\prime}\right|+\left|V^{\prime}\right| \leq 2\left|V^{\prime}\right|$. Therefore, $|V|$ is at most twice the size of a minimum vertex cover of $G$.

Let $s$ be the size of a maximum ocular-free induced subgraph of $G_{u, v, H}$ and $r$ be the size of a minimum vertex cover of $G$. By Lemma 1 item 4 and item $5, s=\left|V\left(G_{u, v, H}\right)\right|-r=|V|+4|E|-r$. Then, by the previous considerations $s=|V|+4 \frac{3}{2}|V|-r \leq 2 r+12 r-r=13 r$. This shows that the first condition for an L-reduction is satisfied with $\alpha=13$.

Given an ocular-free induced subgraph $S=G_{u, v, H}-T$ of $G_{u, v, H}$, the algorithm $g$ of the L-reduction is analogous to the one described in the proof of Lemma 1. It obtains an ocular-free subgraph $S^{\prime}=G_{u, v . H}-T^{\prime}$ of $G_{u, v, H}$, with $T^{\prime}$ a vertex cover of $G$ and $\left|T^{\prime}\right| \leq|T|$. Thus $\left|T^{\prime}\right|-r \leq|T|-r=\left(\left|V\left(G_{u, v, H}\right)\right|-|V(S)|\right)-r=\left(\left|V\left(G_{u, v, H}\right)\right|-r\right)-|V(S)|$. By Lemma 1 item 5, $\left(\left|V\left(G_{u, v, H}\right)\right|-r\right)=s$; then $\left|T^{\prime}\right|-r \leq s-|V(S)|$, which shows that $\beta=1$ suffices to satisfy the second condition for an L-reduction.

\section{A general theorem}

In order to generalize the results of Section 3, we consider, in the present section, general graphs $G$ and $H$, and $u, v$ two non-adjacent vertices of $H$. The $(u, v, H)$-edge-replacing operation of $G$ is the one which obtains the graph $G_{u, v, H}$ where $V(G) \subseteq V\left(G_{u, v, H}\right)$, and for each edge $x y$ of $G$ there is a copy $S_{x y}$ of $H-\{u, v\}$ in $G_{u, v, H}$, and the additional set of edges

$$
E_{x y}=\left\{x s, y t: s, t \in V\left(S_{x y}\right) \text { and } u s, v t \in E(H)\right\} .
$$

For each edge $x y$ of $G$, denote by $H_{x y}$ the subgraph of $G_{u, v, H}$ induced by $V\left(S_{x y}\right) \cup\{x, y\}$. Notice that $H$ and $H_{x y}$ are isomorphic, i.e. $G_{u, v, H}$ contains $H$ as an induced subgraph.

Observe that the graph $G_{u, v, H}$ obtained may depend on the order in which the end vertices of each edge $x y$ of $G$ are considered. However, the properties of $G_{u, v, H}$ used in the proof of the following results hold regardless of whether we consider for an edge $x y$ of $G$ the order $x, y$ or the order $y, x$.

Theorem 4. Let $A$ be a class of graphs characterized by a collection $\mathcal{F}$ of forbidden induced subgraphs satisfying that there exists a member $H$ of $\mathcal{F}$, with a pair of non-adjacent vertices $u$ and $v$, such that, for every graph $G$, the only induced subgraphs of $G_{u, v, H}$ isomorphic to any element of $\mathscr{F}$ are the subgraphs $H_{x y}$, where $x y$ is any edge of $G$. Then, for any graph $G$,

$$
O p t_{\mathrm{MAX}-\mathcal{A}}\left(G_{u, v, H}\right)=n+m(|V(H)|-2)-O p t_{\mathrm{MIN}-\mathrm{vC}}(G),
$$

where $n=|V(G)|$ and $m=|E(G)|$.

Proof. Clearly, $\left|V\left(G_{u, v, H}\right)\right|=n+m(|V(H)|-2)$. We prove first that

$$
\operatorname{Opt}_{\mathrm{MAX}-\mathcal{A}}\left(G_{u, v, H}\right) \geq n+m(|V(H)|-2)-O p t_{\mathrm{MIN}-\mathrm{vc}}(G) .
$$


Let $V^{\prime}$ be a feasible solution of MIN-VC for the instance $G$, with cost $\left|V^{\prime}\right|$. The removal of the same vertices of $V^{\prime}$ from $G_{u, v, H}$ produces a feasible solution $S$ of MAX-A for the instance $G_{u, v, H}$, with $\operatorname{cost} n+m(|V(H)|-2)-\left|V^{\prime}\right|$. Thus, $O p t_{\text {MAX-A A }}\left(G_{u, v, H}\right) \geq n+m(|V(H)|-2)-\left|V^{\prime}\right|$. If in particular $V^{\prime}$ is any optimal solution, then $\left|V^{\prime}\right|=O p t_{\mathrm{MIN}-\mathrm{vc}}(G)$ and the proof follows. Now we prove that

$$
O p t_{\text {MAX-A }}\left(G_{u, v, H}\right) \leq n+m(|V(H)|-2)-O p t_{\text {MIN VC }}(G)
$$

Consider a feasible solution $S$ of MAX-A for $G_{u, v, H}$ and assume that there exists an edge $x^{\prime} y^{\prime}$ of $G$ such that both end vertices $x^{\prime}$ and $y^{\prime}$ belong to $S$. Then, since $A$ is characterized by being $\mathcal{F}$-free and the only induced subgraphs of $G_{u, v, H}$ isomorphic to an element of $\mathcal{F}$ are the subgraphs $H_{x y}$ induced by $V\left(S_{x y}\right) \cup\{x, y\}$, where $x y$ is any edge of $G$, at least one vertex $h$ of $S_{x^{\prime}, y^{\prime}}$ does not belong to $S$. Then, by adding vertex $h$ to $S$ and by removing either vertex $x^{\prime}$ or vertex $y^{\prime}$, another feasible solution of MAX- $A$ for $G_{u, v, H}$, with equal size to $S$, is obtained.

Hence we can assume that $V\left(G_{u, v, H}\right)-S$ contains a vertex cover $V^{\prime}$ of $G$. Thus $V^{\prime}$ is a feasible solution of MiN-vc for $G$ with size $\left|V^{\prime}\right| \leq\left|V\left(G_{u, v, H}\right)-S\right|=n+m(|V(H)|-2)-|S|$; thus Opt $t_{\text {MIN-vc }}(G) \leq n+m(|V(H)|-2)-|S|$. In particular, if $S$ is an optimal solution of MAX-A then $|S|=O p t_{\text {MAX }-A}\left(G_{u, v, H}\right)$; and the proof follows.

Theorem 5. If A is a class of graphs satisfying the conditions of Theorem 4, then MAX-A is Max SNP-hard.

Proof. We L-reduce the Max SNP-hard problem MIN-VC restricted to planar cubic graphs $[13,11,10]$ to MAX-A.

Let $G=(V, E)$ be an instance of MIN-VC restricted to planar cubic graphs. Remark that

$$
|E|=\frac{3}{2}|V| \text { and }|V| \leq 20 p t_{\mathrm{MIN}-\mathrm{vc}}(G) .
$$

Let $u, v$ and $H$ be respectively the two vertices and the forbidden induced subgraph for class $A$ considered in the hypothesis of Theorem 4. The size of graph $H$ is a constant; it is independent from the size of $G$.

The algorithm $f$ of the L-reduction is the one which obtains from $G$, in polynomial time, the instance $G_{u, v, H}$ for Max-A.

By Theorem 4 and (1) above with $|V|=n$ and $|E|=m$.

$$
\begin{aligned}
O p t_{\mathrm{MAX}-\mathcal{A}}\left(G_{u, v, H}\right) & =n+m(|V(H)|-2)-O p t_{\mathrm{MIN}-\mathrm{VC}}(G) \\
& =n+\frac{3}{2} n(|V(H)|-2)-O p t_{\mathrm{MIN}-\mathrm{VC}}(G) \\
& =n\left(\frac{3}{2}|V(H)|-2\right)-O p t_{\mathrm{MIN}-\mathrm{VC}}(G) \\
& \leq 2 O p t_{\mathrm{MIN}-\mathrm{VC}}(G)\left(\frac{3}{2}|V(H)|-2\right)-O p t_{\mathrm{MIN}-\mathrm{VC}}(G) \\
& =(3|V(H)|-5) O p t_{\mathrm{MIN}-\mathrm{Vc}}(G) .
\end{aligned}
$$

This shows that $\alpha=(3|V(H)|-5)$ suffices to satisfy the first condition for an L-reduction.

Given a feasible solution $S$ of MAX-A for the instance $G_{u, v, H}$, with cost $c^{\prime}=|S|$, the algorithm $g$ of the L-reduction is the one which obtains the feasible solution $V^{\prime}$ of MIN-Vc for the instance $G$, with cost $c=\left|V^{\prime}\right| \leq n+m(|V(H)|-2)-c^{\prime}$, as described in the proof of Theorem 4 . Then,

$$
\begin{aligned}
\left|c-O p t_{\mathrm{MIN}-\mathrm{Vc}}(G)\right| & =c-O p t_{\mathrm{MIN}-\mathrm{vc}}(G) \\
& \leq n+m(|V(H)|-2)-c^{\prime}-\left(n+m(|V(H)|-2)-O p t_{\mathrm{MAX}-\mathcal{A}}\left(G_{u, v, H}\right)\right) \\
& =-c^{\prime}+O p t_{\mathrm{MAX}-\mathcal{A}}\left(G_{u, v, H}\right)=\left|c-O p t_{\mathrm{MAX}-\mathcal{A}}\left(G_{u, v, H}\right)\right| .
\end{aligned}
$$

Hence, $\beta=1$ suffices to satisfy the second condition for an L-reduction.

We mention diamond-free, gem-free, and $K_{3.3}$-free graphs as other classes of graphs for which these results are applicable.

\section{Two polynomial approximations}

In this section we first consider the maximization problems MAX-H $\mathcal{K}$, MAX- $\mathcal{H}$ and MAX- $\mathcal{K}$ restricted to graphs with bounded degree, followed by a polynomial 6-approximation algorithm, to minimize the number of vertices to be removed in order to obtain a hereditary clique-Helly subgraph.

\subsection{An approximation algorithm for $\mathrm{MAX}-\mathrm{H} . \mathcal{K} H, \mathrm{MAX}-\mathcal{K} H$, and $\mathrm{MAX}-\mathcal{K}$ restricted to a graph with bounded vertex degree}

In this section we consider the maximization problems $\mathrm{MAX}-\mathrm{H} \mathcal{K} \mathrm{H}, \mathrm{MAX}-\mathcal{K} \mathrm{H}$ and $\mathrm{max}-\mathcal{K}$ restricted to graphs with bounded degree.

We claim that if the input graphs have fixed maximum degree $\Delta$, then there is a polynomial-time $\frac{1}{4+1}$-approximation algorithm for these problems. 
Indeed, let $G=(V, E)$ be a graph with maximum degree at most $\Delta$. Observe that an independent set $S$ of $G$ can be greedily extracted in polynomial time from $V$ by recursively adding a vertex $v$ to $S$ and removing $v$ and $N(v)$ from $V$. Notice that $|V| \leq(\Delta+1)|S|$.

Since an independent set $S$ is at the same time a clique, a clique-Helly and a hereditary clique-Helly subgraph of $G$, we have obtained a feasible solution of size $|S| \geq \frac{|\mathrm{V}|}{\Delta+1}$.

The approximation ratio $\rho$ of the algorithm is the relation between the size of the given solution and the optimal solution; since the optimal solutions have size at most $|V|$, then

$$
\rho \geq \frac{\frac{|V|}{\Delta+1}}{|V|}=\frac{1}{\Delta+1}
$$

Notice that if $A$ is a class of graphs characterized by a collection $\mathscr{F}$ of forbidden induced subgraphs satisfying the conditions of Theorem 4, and no member of $\mathscr{F}$ is an independent set of vertices, then the previous algorithm can be also applied to approximate MAX-A restricted to graphs with bounded vertex degree.

\subsection{A 6-approximation algorithm for $\mathrm{H} \mathcal{K} H$}

In this section we consider the following minimization problem: given a graph $G=(V, E)$, find a minimum subset $V^{\prime} \subset V$ such that $V-V^{\prime}$ induces a hereditary clique-Helly subgraph of $G$. Since the class $\mathrm{H} \mathcal{K} H$ is characterized by forbidding an ocular graph as induced subgraph, we can design a 6-approximation algorithm $\Pi$ by recursively looking for an ocular induced subgraph in the current graph; in the case that we find, its six vertices are removed from the current graph.

Each ocular induced subgraph found requires at least one vertex in the optimum solution. As all of them are vertex disjoint, we have taken at most six times the size of the optimum solution.

\section{Concluding remarks and future work}

We summarize in the following table our contribution to $\mathrm{H} \mathcal{K}, \mathcal{K} \mathrm{H}$ or $\mathcal{K}$ and the ones in the literature.

\begin{tabular}{|c|c|c|c|}
\hline Class & Recognition & Induced subgraph & Maximum induced subgraph \\
\hline \multirow[t]{2}{*}{ Clique graph } & $\mathcal{K}-\mathrm{REC}$ & $\mathcal{K}$-SUB & $\operatorname{MAX}-\mathcal{K}$ \\
\hline & NPC [9] & NPC & Max SNP-hard \\
\hline \multirow[t]{2}{*}{ Clique-Helly } & KH-REC & $\mathcal{K H}$-SUB & $\mathrm{MAX}-\not \mathcal{H}$ \\
\hline & $\mathbf{P}[8]$ & NPC & Max SNP-hard \\
\hline Hereditary & $\mathrm{H} \mathcal{K} H$-REC & н $\mathcal{K} H$-SUB & MAX-H $\mathcal{K} \mathrm{H}$ \\
\hline clique-Helly & $\mathbf{P}[7]$ & $\mathbf{N P C}[6]^{*}$ & Max SNP-hard [14] \\
\hline
\end{tabular}

In the present paper for maximum degree 6 graphs.

We observe, as a property derived from the reduction, that the results in $[6,14]$ prove, respectively, that $A$-A-SuB is NPcomplete, and that MAX-A is Max SNP-hard for graphs with maximum degree at least 32, while Theorem 4 propose an alternative classification for a hereditary property, classifying .A-SUB as NP-complete and MAX-A as Max SNP-hard for graphs with maximum degree bounded by at most three times the maximum degree of $H$.

We have proved that if $A$ is $\mathrm{H} \mathcal{K} H, \mathcal{K} H$ or $\mathcal{K}$ then $A$-SUB is NP-complete and MAX-A is Max SNP-hard for maximum degree 6 graphs. However, since any ocular graph has a four-degree vertex, all these problems are polynomial for maximum degree 3 graphs. Hence, it is left as an open problem to determine the maximum $k, 3 \leq k \leq 5$, such that $A$-suB or MAX-A is a polynomial problem for maximum degree $k$ graphs. Besides, we are currently working on the design of approximation algorithms for the problems $\operatorname{MAX}-\mathcal{K}, \operatorname{MAX}-\mathcal{K} \mathrm{H}$, and $\operatorname{MAX}-\mathrm{H} \mathcal{K} \mathrm{H}$.

\section{Acknowledgments}

We are grateful to the referees for their careful reading which helped to improve the paper.

\section{References}

[1] M.C. Dourado, F. Protti, J.L Szwarcfiter, Computational aspects of the Helly property: A survey, J. Braz. Comput. Soc. 12 (2006) 7-33.

[2] A. Brandstâdt, V.B. Le, J.P. Spinrad, Graph classes: A survey, in: SIAM Monographs on Discrete Mathematics and Applications, 1999.

[3] T.A. McKee, F.R. McMorris, Topics in intersection graph theory, in: SIAM Monographs on Discrete Mathematics and Applications, 1999.

[4] E. Prisner, Graph Dynamics, in: Pitman Research Notes in Mathematics, vol. 338, Longman, 1995.

[5] J.L. Szwarcfiter, A survey on clique graphs, in: C. Linhares-Sales, B. Reed (Eds.), Recent Advances in Algorithmic Combinatorics, Springer-Verlag, 2002.

[6] J. Lewis, M. Yannakakis, The node-deletion problem for hereditary properties is NP-complete, J. Comput. System Sci. 20 (2) (1980) $219-230$.

[7] E. Prisner, Hereditary clique-Helly graphs, J. Combin. Math. Combin. Comput. 14 (1993) 216-220.

[8] J.L. Szwarcfiter, Recognizing clique-Helly graphs, Ars Combin. 45 (1997) 29-32. 
[9] L. Alcón, L. Faria, C.M.H. de Figueiredo, M. Gutierrez, Clique graph recognition is NP-complete, in: Proc. WG 2006, in: Lecture Notes in Comput. Sci., vol. 4271, Springer, 2006, pp. 269-277.

[10] C.H. Papadimitriou, M. Yannakakis, Optimization, approximation, and complexity classes, J. Comput. System Sci. 43 (1991) 425-440.

[11] S. Arora, C. Lund, R. Motwani, M. Sudan, M. Szegedy, Proof verification and hardness of approximation problems, in: Proc. IEEE Symposium on Foundations of Computer Science, FOCS'92, 1992, pp. 14-23.

[12] R. Karp, Reducibility among combinatorial problems, in: R.E. Miller, J.W. Thatcher (Eds.), Complexity of Computer Computations, Plenum Press, New York, 1972, pp. 85-103.

[13] P. Alimonti, V. Kann, Hardness of approximating problems on cubic graphs, in: Workshop ASHCOMP'96, Udine, Italy, September 10-19, pp. $288-298$.

[14] C. Lund, M. Yannakakis, The approximation of maximum subgraph problems, in: Proc. 20th Int. Colloquium on Automata, Languages and Programming, in: Lecture Notes in Comput. Sci., vol. 700, Springer-Verlag, pp. 40-51. 